\title{
Adequacy Assessment of Future Electricity Networks
}

\author{
W. A. Bukhsh, G. S. Hawker, K. R. W. Bell \\ Institute for Energy and Environment, \\ Department of Electronic and Electrical Engineering, \\ University of Strathclyde, Glasgow, United Kingdom. \\ $\{$ waqquas.bukhsh, graeme.hawker, keith.bell\}@ @strath.ac.uk
}

\author{
Tim Bedford \\ Department of Management Science, \\ University of Strathclyde, \\ Glasgow, Scotland. \\ tim.bedford@strath.ac.uk
}

\begin{abstract}
Liberalisation of electricity markets, changing patterns in the generation and use of electricity and new technologies are some of the factors that result in increased uncertainty about the future operating conditions of our power system. In this context, planning for future investments in power system requires careful selection and assessment of future operating conditions. This paper revisits the notion of power system adequacy and highlights the need for consideration of some factors that have hitherto tended not to be part of a transmission expansion planning process, in particular in respect of the credible range of possible values of system operating conditions and transitions between successive operating states. Firstly, we present some definitions of power system operational regions. Secondly, we present a stochastic optimisation model that measures the adequacy of a transmission network for given future operating conditions. Uncertainties in demand and generation are modelled using a large number of scenarios. The optimisation model identifies the critical future operating conditions needing the special attention of a power system planner. The proposed model is simulated on a 39-bus network, whereby it is shown that this model can identify critical operating conditions that need the attention of a system planner.
\end{abstract}

Index Terms - adequacy assessment; transmission planning; renewable energy sources; stochastic optimisation.

\section{INTRODUCTION}

Modern power systems are in the midst of a comprehensive change, initially driven by liberalisation of electricity markets and now with an increased focus on renewable energy sources (RES). Over the last decade, there has been a substantial increase in installed capacities of RES and in some countries instantaneous penetration of power from RES have reached levels upto $50 \%$ of generation [1]. This paradigm shift has introduced new challenges for transmission system operation and planning [2]. Now power system planners need to consider the uncertainties associated with the evolution of demand, fuel prices, generation entry/closures whilst also needing to take into account the operational uncertainties stemming from RES. All these aforementioned uncertainties have temporal as well

The research leading to these results has received funding from the European Union Seventh Framework Programme under the project titled GARPUR, Grant Agreement No 608540. as spatial components and a careful consideration of associated correlations is required.

The first step in planning for the future power system is to check the adequacy and security of the future power system. In utilities, this is normally done by solving sets of load flow calculations on a few foreseeable future system operating conditions [3]. Given the complexity of a power system transmission expansion problem (TEP), only a reduced number of future snapshots are considered for analysis. In such a analyses, snapshots of cases that tend towards a worst case identify problems with the future system operation and an expert's judgement is used to come up with a set of expansion plans. This type of analysis does not take into account all the relevant uncertainties or their probability distributions.

\section{A. Academic literature}

A TEP is a challenging problem, primarily because the future conditions for which a system should be planned are increasingly uncertain, particularly over the decadal timescales sometimes considered in transmission investment. In formulations presented in the academic literature, a TEP is usually framed as a mixed integer linear programming problem where investment decisions are modelled using binary variables. Solution of a TEP problem proposes major topological changes that are capital intensive and must translate into increased reliability and/or savings in the cost of operating the future power system, while minimising the risk of stranded assets. Due to the complexity associated with the TEP problem, only a limited number of initial conditions is typically considered in TEP problems.

In this context, there has been significant interest in addressing several aforementioned challenges. A generic framework for a probabilistic TEP problem is proposed in [4], where a step by step approach for assessing candidate decisions for future investments in power systems is proposed. Authors in [5] propose a three level TEP problem where the objective is to minimise the system investment cost plus the worst possible operational cost. Uncertainty is modelled as a robust set that gives worst realisation of uncertain parameters that are bounded by an uncertainty set. A Monte-Carlo scheme is presented in [6] that studies the effect of wind power uncertainty on power system adequacy. A TEP model is 
presented in [7], where the first step is to check the need for investment based on a deterministic criterion. All of the TEP problems depend on a choice of initial conditions about the future operating states and it is not very clear how such initial conditions are obtained. Gaps in the state of the art as represented in the academic literature include:

- a limited number of future snapshots are considered for planning studies and it is not clear how the initial conditions are selected for such snapshots;

- little or no effort is made to check operability of the system during transitions from one time step to another.

\section{B. Our contributions}

It is difficult to accurately predict the future operating state of a power system for a particular time in future. However, it is possible to construct a set of credible operating states. Given the combinatorial number of possibilities for future operating states, the set of credible states can be very large. In this paper, we present a mechanism that can reduce this set to a few operating states that needs the attention of a system planner. The proposed method is based on a stochastic optimisation model that checks the operability of a power system while taking into account the probability of an operating state and impacts in terms of loss of load. We consider the uncertainties that can arise during the future operation of a power system and evaluate how power system adequacy metrics are affected by these uncertainties. The proposed methodology identifies operating states that need the attention of a transmission system planner and we characterise them as critical operating states. The critical operating states may have arisen due to nonoperability of the future power system or due to operability of the future system but at a very high cost. This identifies a need for investment in the former case 'reliability driven' and, in the latter, 'economy driven', and the system planner can then use expert judgement or further analysis to determine the type and size of an investment. The contributions of our approach are fourfold:

1) a tractable formulation that accounts for temporal considerations and a large number of scenarios for the future power system operation;

2) a method for identifying and ranking the critical operating states for further analysis;

3) improved adequacy assessment for future power systems with high penetration levels of RES;

4) a highlighting of need to consider not only snapshots but also the transitions between snapshots.

The remainder of the paper is organised as follows. Section II describes the notion of adequacy assessment in power systems and gives details of the uncertainties involved in adequacy assessment of future power systems. Section III gives the conceptual framework of the adequacy assessment method. Section IV formulates the stochastic optimisation approach for adequacy assessment and in section $\mathrm{V}$ we give numerical examples of the proposed method. Conclusions and future research directions are given in section VI.

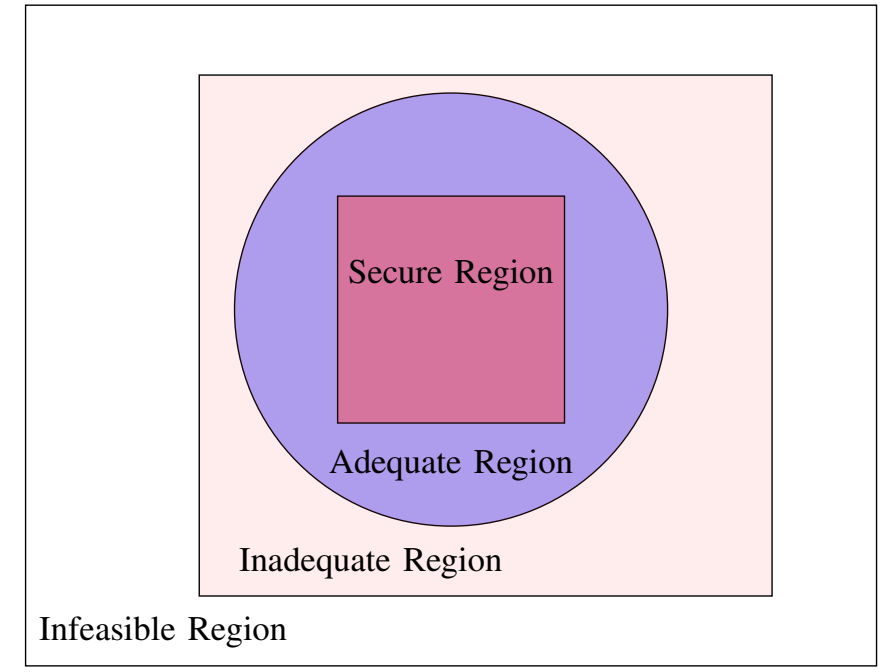

Figure 1. Operability regions of a power system.

\section{AdEQUACY ASSESSMENT OF THE FUtURE NETWORK}

Power systems are operated under a certain security criterion that in most cases means no contingency from within a particular defined set should endanger the supply of power to the end users. Following a contingency, the operating point could be pushed to an unsecured region and an intervention may be required from a system operator to re-secure the operating state of a system. Fig. 1 shows four operability regions of a power system, where the innermost region is called the secure region and most of the time an operating point of a power system should lie within this region. An operating point is in the adequate region if it is not secure but the system is able to meet all the demand whilst respecting network and physical constraints. If a system is only able to meet part of the total demand then the operating point lies in an inadequate region.

The infeasible region represents a situation of a wide-area blackout, where a black-start is required to revive the system. Within the infeasible region, the power flow equations do not have a real solution, whereas in the inadequate region a real power flow solution exists. Following we give definitions of adequate operating point and secure operating point as follows.

Definition 1 (Adequate operating point). A power system state where the system continues to supply all demand but any of the security limits might be violated; meaning that any contingency from within a defined security set would result in either loss of supply and/or violation of transmission constraints.

Definition 2 (Secure operating point). A power system state where the system is able to supply the aggregate electric power and energy requirement of all the customers and it is able to do so after the occurrence of any one contingency within a given defined set, e.g. all the $(N-1)$ events.

The region spanned by all adequate operating points is called an adequate region and the region spanned by the 
secure operating points is called a secure region, as shown in the Fig. 1. Further characterization of a secure operating point can be found in [8]. An example of a defined set of contingencies, i.e. of 'secured events', can be found in [9]. The following lemma states an obvious relationship between system adequacy and system security.

Lemma 1 (Relationship between adequacy and security). If a power system is secure then it is adequate.

Proof. The proof follows from the definitions of power system adequacy and power system security.

From lemma 1 we note that power system adequacy is a necessary condition for power system security. In this paper, we restrict our analysis to determining adequacy of the future power system. Further analysis is required to check if an adequate operating state is secure.

\section{A. Uncertainties involved in the adequacy assessment}

In the day-ahead or the hour-ahead operation of a power system, the objective of a system operator is to minimise total operational costs while maintaining an adequate level of reliability. In these time scales, the values of various random variables can be predicted with reasonable accuracy and, if the system has been sufficiently well planned, can be managed in the real time operation of a power system. However when system operation is seen years in advance, the framework for analysis needs to adapt and it is inevitable to consider a large set of credible future operating states that capture various uncertainties. For example, day-ahead demand in the Great Britain (GB) system can be predicted to good accuracy (e.g. see demand forecast and out-turn values in [10]). However, it is very difficult to say something about the demand of a particular day in the year 2019.

Figure 2 shows a fan chart describing evolution of peak electricity demand for the GB transmission system under four different scenarios. The forecasts are obtained from future energy scenarios from the GB system operator, National Grid [11], whereas the inner graphs are based on historic data obtained from [10]. The chart fans out as we look further into the future because we are less and less certain about the possible operating conditions in the future.

The yearly scenarios in Fig. 2 show a difference of approximately $10 \mathrm{GW}$ in peak demand in the year 2034/35. If we cluster the yearly demand for the year 2013/14 according to seasons, we note an absolute demand variation of $31.4 \mathrm{GW}$ in the winter of 2013/14 between the highest evening peak and lowest overnight minimum. If we further look into individual days of the winter of 2013/14, we note a maximum absolute variation of $15 \mathrm{GW}$ over any single day. This shows that only taking a snapshot of demand for a season or a year is not sufficient to capture the underlying uncertainty in demand.

Demand is only one of the many uncertainties that a system planner needs to take into account whilst planning for the future power system. Generation from RES is another uncertainty that must be considered for adequacy assessment of a power system and subsequent security assessment. Asset condition, generator openings/closures, and long duration equipment outages, whether planned (for maintenance or construction work) or unplanned are some other uncertainties that affect the adequacy of power system. In this paper, we only consider the uncertainties stemming from demand and RES generation during the future operation of power system. However, the approach proposed in this paper can be extended to account for other uncertainties.

\section{B. Scenarios for adequacy assessment}

Forecasting for future credible states of a power system is an independent area of research and has attracted a lot of attention. In this paper, we consider the operational scenarios for demand and generation from RES as an input to our stochastic optimisation model. Publicly available generation and demand 'macro-scenarios', i.e the background of installed generation capacity and peak demand, for the GB network are obtained from [11] and the operational scenarios for demand and RES are based on the historic data obtained from [10].

Let $\mathscr{S}^{\mathrm{W}}$ denote the set of operating scenarios for RES and $\mathscr{S}^{\mathrm{D}}$ denote the set of operational scenarios for demand, respectively. For ease of presentation we denote a single index set $\mathscr{S}$ for scenarios. The set $\mathscr{S}$ is defined as:

$$
\mathscr{S}:=\left\{s=\left(s_{d}, s_{w}\right): s_{d} \in \mathscr{S}^{\mathrm{D}}, s_{w} \in \mathscr{S}^{\mathrm{W}}\right\}
$$

Let $P_{w, s, t}^{\mathrm{W}}$ be the available generation and $p_{w, s, t}^{\mathrm{W}}$ be the real power generation injection at the node $w$ during the time period $t$, respectively. The RES power output for the generator $w$ is modelled using the following equation:

$$
0 \leq p_{w, s, t}^{\mathrm{W}} \leq P_{w, s, t}^{\mathrm{W}}
$$

\section{CONCEPTUAL FRAMEWORK}

Our conceptual framework is as follows: a system planner is faced with the possibility that a credible future operating state will be insecure or, worse, inadequate. When the time comes, the system operator should intervene to change the operating state to make it secure (with the action very likely to incur a cost) or, if they do not, it is because insufficient means are available to the system operator to do so. In either case, the transmission expansion planner should consider whether investment in additional network facilities are required to render the system securable under those circumstances or reduce the cost of the operator's intervention. Following on from Lemma 1, a first step is determine whether the credible future operating state is adequate. There is an uncertainty in the future about demand and generation, and that uncertainty is captured by a large number of macro-scenarios and, for each of them, a large number of operating scenarios. For a given macro-scenario, the system planner wants to determine the scenarios containing critical operating conditions that require more detailed assessment.

The TEP problem can be posed as a two-stage stochastic program. The first stage of the problem could model the 


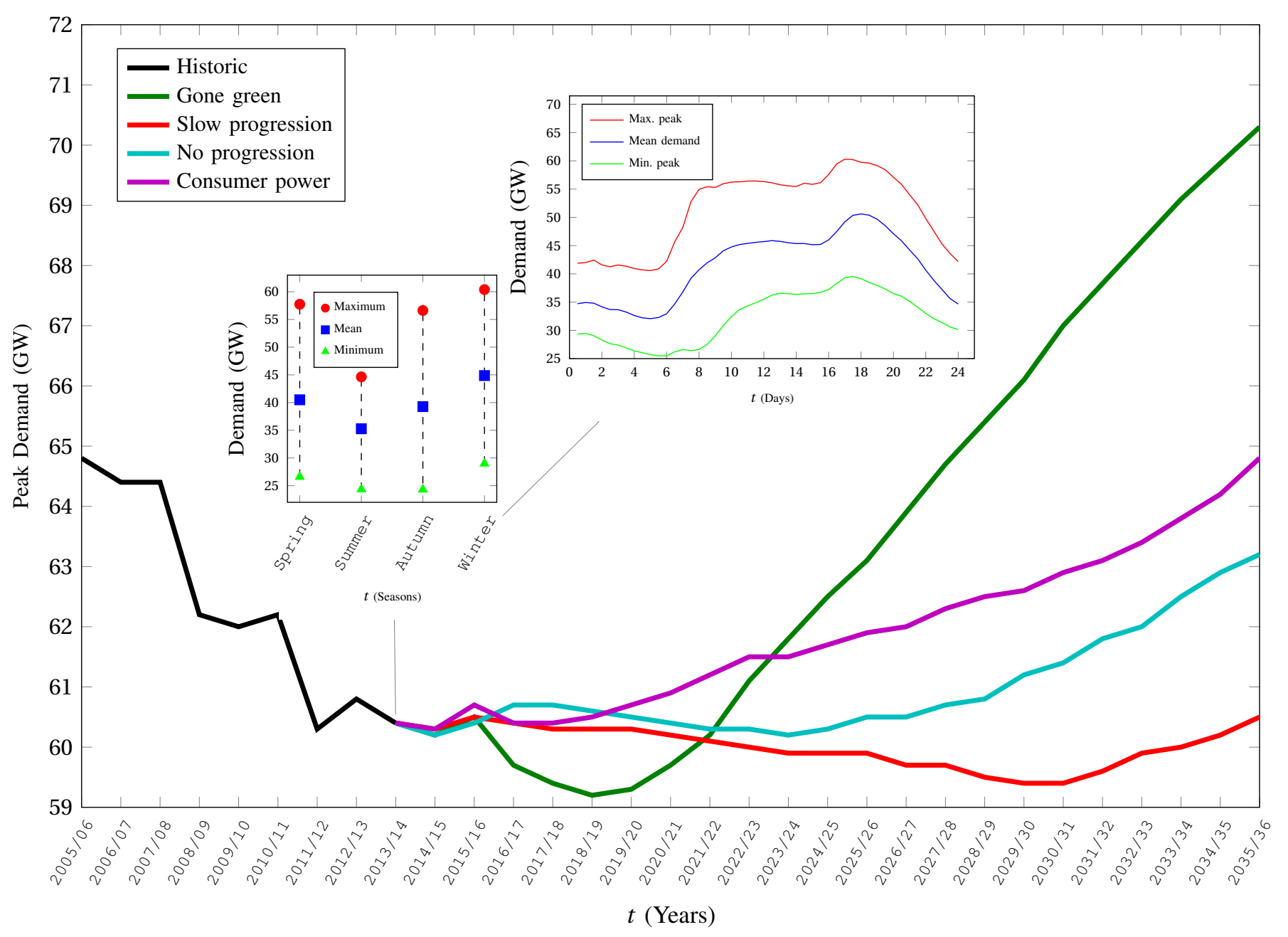

Figure 2. Demand forecast for the GB network. Four different scenarios for years upto 2035 are taken from [11]. Small figures show seasonal and daily load curves for the year 2013/14.

transmission expansion candidates. The second stage realises the operation of the power system and the actions that may be taken by a system operator, such as rescheduling generation and shedding load. In this paper, we fix the first decision variables to the given topology of the network and study the impact of future system operation on the adequacy. Inadequacy in future operation of power system will identify a need for investment.

The general form of a two stage stochastic program [12] is given as:

$$
\min c^{\mathrm{T}} x+E_{\xi} Q(x, \xi)
$$

subject to

$$
\begin{gathered}
A x=b, \\
x \geq 0,
\end{gathered}
$$

where $Q(x, \xi)=\min \left\{q^{\mathrm{T}} y: W y=h-T x, y \geq 0\right\}$ is the optimal value of the second stage problem.

In the TEP problem, the values of the first stage decision variables determine the network facilities available to the system operator. These influence the operability of the system and the cost of operation evaluated at the second for a range of operating scenarios. The first stage may include a random vector representing variations in the 'macro-scenario', i.e. installed generation and demand growth. The random vector $\xi$ defines the uncertainty in future demand and RES generation, $y$ is a vector of the second-stage variables that model second stage actions like generation rescheduling and load shedding. The second stage decisions are implemented on the realisation of the random vector $\xi$. In the next section, we give the mathematical formulation of the problem.

\section{PROBLEM FORMULATION}

Consider an electricity network $\mathscr{N}$ that comprises of a set of buses $\mathscr{B}=\left\{1,2, \cdots, n^{\mathrm{B}}\right\}$ and a set of lines $\mathscr{L}$. Let a set of generators be $\mathscr{G}$ and a set of loads $\mathscr{D}$. A subset $\mathscr{G}_{b}$ of generators is attached to a bus $b \in \mathscr{B}$; similarly, $\mathscr{D}_{b}$ is the subset of loads and $\mathbb{W}_{b}$ is a subset of RES generators connected at a bus $b \in \mathscr{B}$, respectively. 


\section{A. Power flow}

In this paper, we use a well know DC approximation of nonlinear power flow equations, in which series resistance and shunt admittances are neglected, voltage magnitudes are assumed to be equal to 1.0 per unit and small angle approximations are used [13]. This approximation ignores voltages and reactive power. We note that shortfalls or excesses of reactive power can be quite readily addressed in 9-18 month timescales by installation of sufficient reactive compensation in appropriate locations. On the other hand, enhancements of the network's thermal capacities can take many years, often because of issues around obtaining planning consents, and hence must be identified well in advance and therefore form the core of the expansion planner's task. As a consequence, in this work, we concentrate on thermal network constraints which can be approximated quite well by the 'DC' power flow equations and give significant computational advantages.

Let $p_{g, s, t}^{\mathrm{G}}$ be the real power generation from a conventional generator $g$ in a time interval $t$ if an operating scenario $s$ is realised. The power balance equations are given as, $\forall b \in$ $\mathscr{B}, s \in \mathscr{S}, t \in \mathscr{T}$ :

$$
\sum_{g \in \mathscr{G}_{b}} p_{g, s, t}^{\mathrm{G}}+\sum_{w \in \mathscr{W}_{b}} p_{w, s, t}^{\mathrm{W}}=\sum_{d \in \mathscr{D}_{b}} p_{d, s, t}^{\mathrm{D}}+\sum_{l \in \mathscr{L}_{b}} p_{l, s, t}^{\mathrm{L}}
$$

where $p_{d, s, t}^{\mathrm{D}}$ denotes the real power delivered to the demand $d$ and $p_{l, s, t}^{\mathrm{L}}$ is the flow of real power in the line $l$ in the time period $t$ in the case when scenario $s$ is realised, respectively. The power flow equations are given as, $\forall l \in \mathscr{L}, s \in \mathscr{S}, t \in \mathscr{T}$ :

$$
p_{l, s, t}^{\mathrm{L}}=b_{l}\left(\theta_{b, s, t}-\theta_{b^{\prime}, s, t}\right)
$$

where $b_{l}$ is susceptance, and $b$ and $b^{\prime}$ are the two ends of the line $l$, respectively. Voltage angles at the two ends of the line $l=\left(b, b^{\prime}\right)$ are denoted by $\theta_{b, s, t}$ and $\theta_{b^{\prime}, s, t}$, respectively.

\section{B. Demand Model}

Let $\mathscr{D}$ denote the set of real power demands observed at the exit points of the transmission network, and $P_{d, s, t}^{\mathrm{D}}$ denote the real power demand at bus $d \in \mathscr{D}$. Let $\alpha_{d, s, t}$ be proportion of the demand delivered at bus $d$ at the time period $t$ for the scenario $s$. The demand model is given by the following set of constraints:

$$
\begin{gathered}
p_{d, s, t}^{\mathrm{D}}=\alpha_{d, s, t} P_{d, t}^{\mathrm{D}} \\
0 \leq \alpha_{d, s, t} \leq 1
\end{gathered}
$$

\section{Operational constraints}

Generation from the conventional generators is bounded by the following inequality constraints:

$$
P_{g, t}^{\mathrm{G}-} \leq p_{g, s, t}^{\mathrm{G}} \leq P_{g, t}^{\mathrm{G}+}
$$

where $P_{g, t}^{\mathrm{G}-}, P_{g, t}^{\mathrm{G}+}$ are the lower and the upper bounds on the generation output of the generator $g$ during time period $t$, respectively.
The ability of a conventional generator $g$ to deviate from its operating point in short time scales is limited. The limitation is often expressed as maximum upward and downward ramp rates [14]. Therefore we limit the amount of change in the generation depending on the ramp rate of the individual generators. The ramp rate constraints are given as:

$$
\begin{aligned}
& R_{g, t}^{-} \leq p_{g, t+1}^{\mathrm{G}}-p_{g, t}^{\mathrm{G}} \\
& p_{g, t+1}^{\mathrm{G}}-p_{g, t}^{\mathrm{G}} \leq R_{g, t}^{+}
\end{aligned}
$$

where $R_{g, t}^{+}, R_{g, t}^{-}$are the upward and downward ramp rates for the generator $g$, respectively. For a single generation unit, the ramp rates does not depend on the operating point of the generator but depend on the type of generator, i.e. a generator can ramp up or ramp down, within its stable generation limits, independent of its output. However, if the generation is aggregated and is modelled as a fleet of a particular type at a node than the ramp rates can be approximated by a percentage change from its current operating point. This approximation means that the aggregated generators can be ramped (up or down) depending on the aggregated generationhigher generation would imply more units committed and is able to offer higher flexibility.

The line flow limits are given by the following set of constraints: $\forall l \in \mathscr{L}, t \in \mathscr{T}, s \in \mathscr{S}$ :

$$
-P_{l, t}^{\max } \leq p_{l, s, t}^{\mathrm{L}} \leq P_{l, t}^{\max }
$$

where $P_{l, t}^{\max }$ is the real power capacity limit of the line $l$ during time period $t$. Note that in order to account for the reactive power, that is not modelled, the real power capacity limit can be taken as a certain proportion (less than 1) of the thermal limit expressed in terms of apparent power.

\section{Adequacy measure}

Let $\lambda_{s}$ be the probability of an operating scenario $s$. We define an adequacy measure $a_{d, t}^{\mathrm{D}}$ for a demand $d$ during time period $t$ as:

$$
a_{d, t}^{\mathrm{D}}=\sum_{s \in \mathscr{S}} \lambda_{s} \alpha_{d, s, t}
$$

The adequacy measure $a_{d, t}^{\mathrm{D}}$ is a measure of the capability of a power system to deliver required amount of power at node $d$ while respecting all the constraints.

\section{E. Objective function}

Let $f\left(p_{g, t}^{\mathrm{G}}\right)$ be the cost function for the conventional generator $g$. We define the objective function as:

$$
z=\sum_{d \in \mathscr{D}}\left(1-a_{d, t}^{\mathrm{D}}\right) P_{d, t}^{\mathrm{D}} V_{d, t}^{\mathrm{D}}+\sum_{g \in \mathscr{G}} f\left(p_{g, t}^{\mathrm{G}}\right),
$$

where $V_{d, t}^{\mathrm{D}}$ is the value of lost load at node $d$ during time $t$. The value of lost load is much greater than the cost of generation and therefore the first part of objective function is always dominant. 


\section{F. Adequacy assessment problem}

The overall formulation of the problem is given as follows:

$$
\min \sum_{t \in \mathscr{T}} z\left(a_{d, t}^{\mathrm{D}}, p_{g, t}^{\mathrm{G}}\right)
$$

subject to

$$
\text { (1), }(3-9)
$$

Depending on the objective function, the overall problem is then a linear or a quadratic program (LP or QP). We use CPLEX 12.06 [15] called from a PYOMO [16] model to solve the problem.

The next lemma defines the adequacy of a power system.

Lemma 2 (Adequacy of a power system). A power system is adequate iff

$$
\sum_{t \in \mathscr{T}} \sum_{d \in \mathscr{D}}\left(a_{d, t}^{D}\right)^{*}=n^{D} n^{T}
$$

where $n^{D}$ is the number of demand nodes, $n^{T}$ is the number of time intervals and $\left(a_{d, t}^{D}\right)^{*}$ is value at the optimal solution of problem (11).

Proof. Proof is omitted for space reasons.

\section{NumericAl TESTS}

Consider a 39-bus network as shown in Fig. 3. This test network is a modified version of the New England test network from [17]. We have replaced three fossil fuel generators with the renewable generators at buses 33, 34 and 36, receptively. Total generation capacity in this network is $7367 \mathrm{MW}$ and approximately $24 \%$ of it is from the RES. Peak demand in this network is $6254 \mathrm{MW}$ i.e. the absolute peak demand such that all the variations concern daily peaks that are less than this value.

Figure 4 shows demand and wind components of operating scenarios that we use in our stochastic programming model. Half-hourly time series of total GB system demand and aggregated wind power generation from each day over the Winter of 2014/15 are taken from [18] and, for each halfhour on each day, expressed as a proportion of the single largest half-hourly demand and wind generation value in the set. Each daily time series forms one instance of demand and wind generation in the set of operating scenarios used in the stochastic optimisation.

Let $n^{\mathrm{D}}, n^{\mathrm{W}}$ be the number of demand and wind operating scenarios, respectively. Fig. 5 shows the simulation results for different values of demand and wind scenarios. Note that the choice $n^{\mathrm{D}}=n^{\mathrm{W}}=1$ corresponds to a deterministic case in which, for each half-hour, the mean values of demand and available wind power in that half-hour are used. We note that the system is adequate for the deterministic case. Further, when the number of scenarios is increased(chosen randomly), we see a decrease in the measure of adequacy. In all cases, there is sufficient generation available somewhere on the system to meet demand. Inadequacy, i.e. failure to meet all demand, arises because, in some scenarios, demand

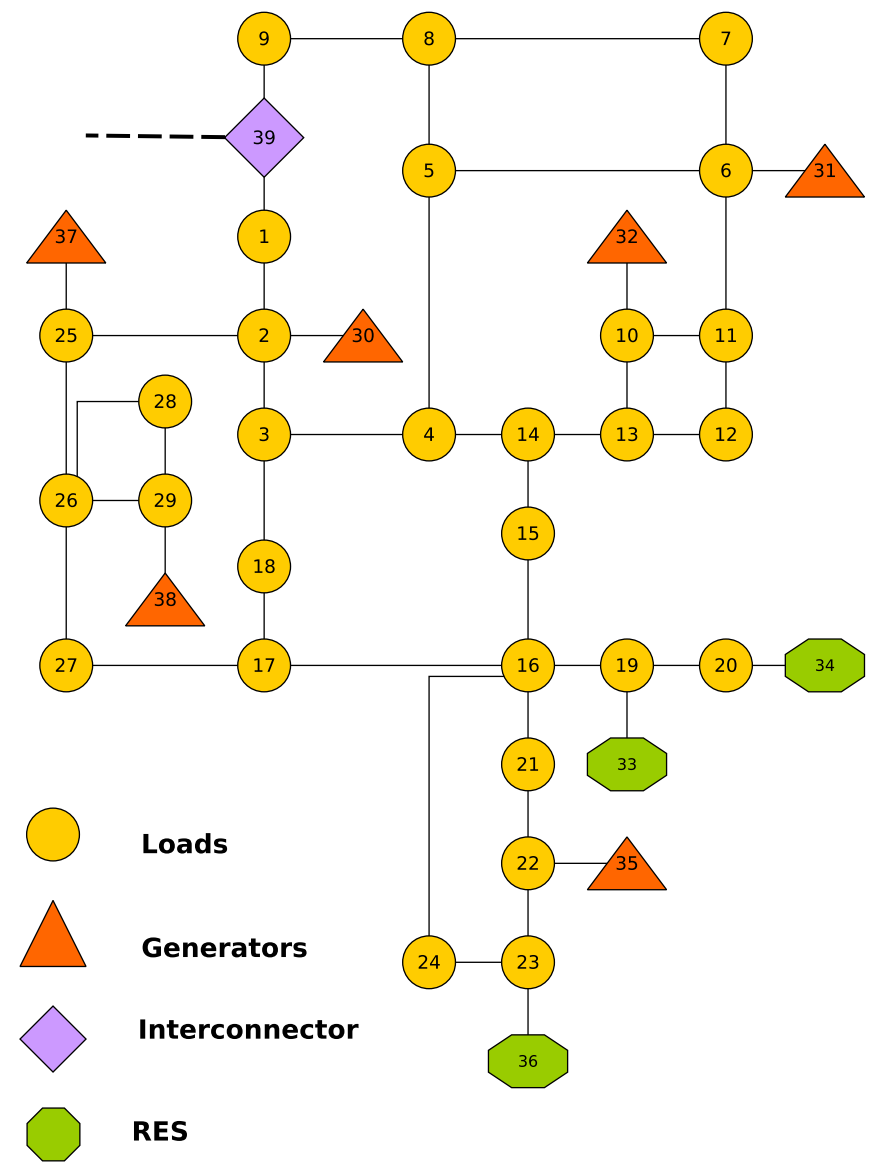

Figure 3. Modified 39 bus system with 6 conventional generators, 3 RES and 1 inter-connector

in an area relative to the power available from RES in that area is high and the network's capacity to import power into that area to meet demand is insufficient. Note that there is no significant difference in the values of adequacy measure for $n^{\mathrm{D}}=15, n^{\mathrm{W}}=30$ and $n^{\mathrm{D}}=50, n^{\mathrm{W}}=100$. In this study, this indicates that $n^{\mathrm{D}}=15, n^{\mathrm{W}}=30$ scenarios are sufficient to capture the underlying uncertainty and adding more scenarios does not have a significant affect on the value of the optimal solution. However, the results are dependent on on which scenarios have been randomly sampled from the full set.

We report the results of a computational search to find critical operating snapshots in Figure 6. We generated 200 random samples for operating scenarios between $1 \leq n^{\mathrm{D}}, n^{\mathrm{W}} \leq 5$, and a snapshot is classified as critical if $a_{d, t}^{\mathrm{D}} \leq 0.995$. Note that $n^{\mathrm{D}}=n^{\mathrm{W}}=1$ corresponds to 48 operating snapshots, as we consider half-hourly time series for each day. Figure 6 shows the min, mean and max number of critical operating states found for a given number of snapshots that were assessed. In each case, the mean number of critical operating snapshots were always less than $10 \%$ of the assessed operating snapshots and the maximum number of critical operating snapshots did not exceed $18 \%$ of the assessed snapshots. We further note that if we relax the condition for classifying critical operating 


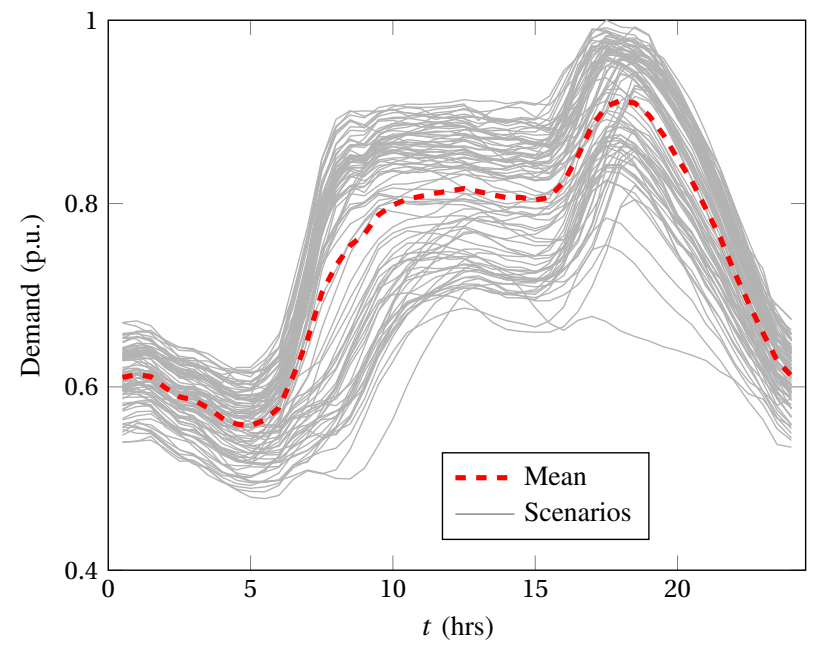

(a) 90 operating scenarios for demand

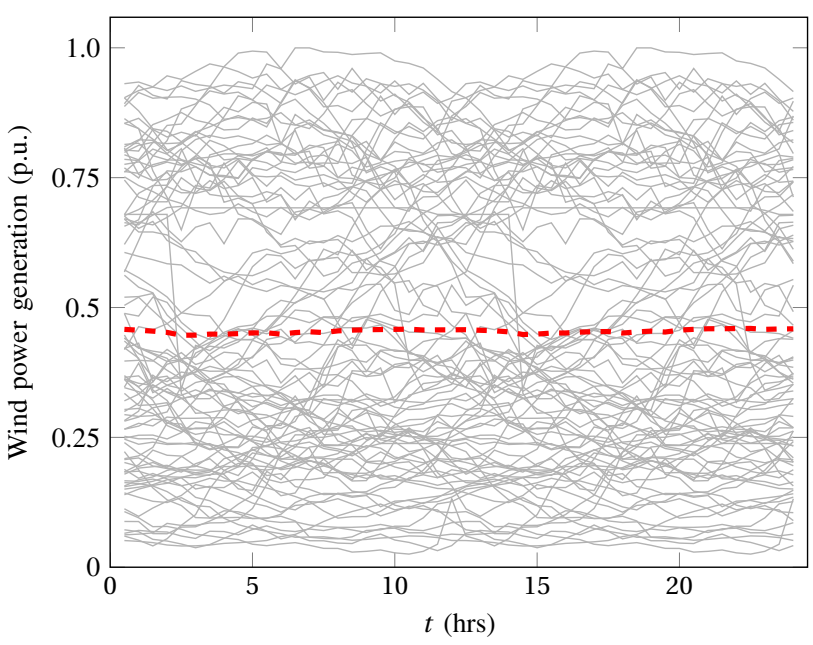

(b) 90 operating scenarios for wind

Figure 4. Operating scenarios for stochastic programming problem. Dashed line show the mean value of all scenarios.

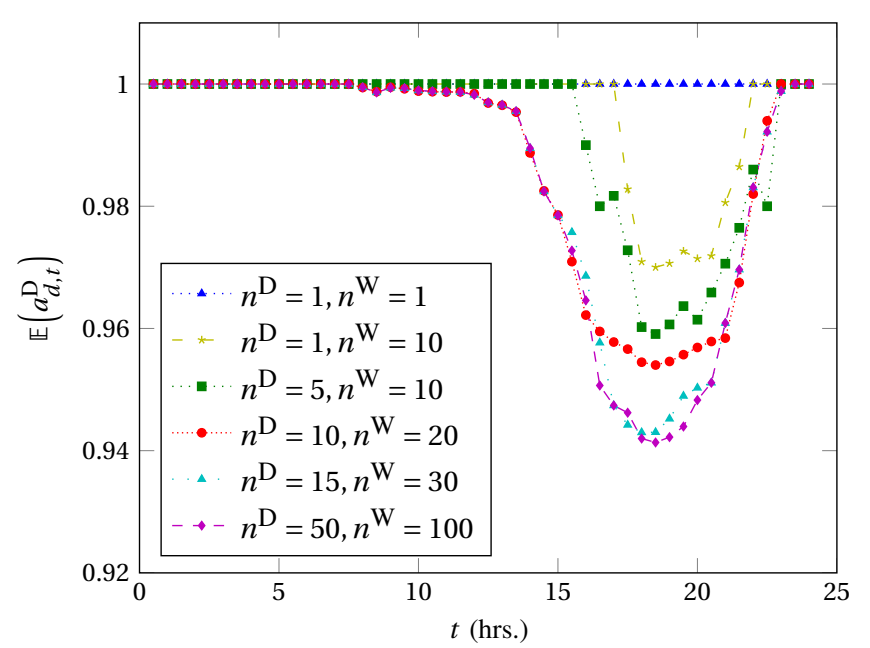

Figure 5. Adequacy accessment of 39 bus network with different number of randomly sampled operating scenarios for demand and available power from RES. Each operating scenario corresponds to a time-series of 48 snapshots.

snapshots to $a_{d, t}^{\mathrm{D}} \leq 0.98$, the mean never exceeded $2.5 \%$ of the accessed operating conditions.

For the next experiment, we fix the number of operating scenarios to $n^{\mathrm{D}}=10, n^{\mathrm{W}}=20$ and, for same set of operating scenarios(which had been randomly sampled from the full set), vary the ramp rate of the conventional generators. The ramp rate constraints are defined in percentage where $R_{g, t}^{ \pm}= \pm x \%$ means that the generators are allowed to ramp-up or rampdown by $x \%$ from their current operating point. Figure 7 shows the results for different ramp rates constraints. We note that the ramp rate constraints are not active for the value of $\pm 20 \%$ i.e. the results are similar to the one shown in Figure 5. The ramp rate constraints are active for the values $R_{g, t}^{ \pm} \leq 12 \%$. Figure 7 shows the affect on the adequacy measure corresponding to three different ramp rate constraints. As expected the affect of ramp rate constraints are at the morning and evening

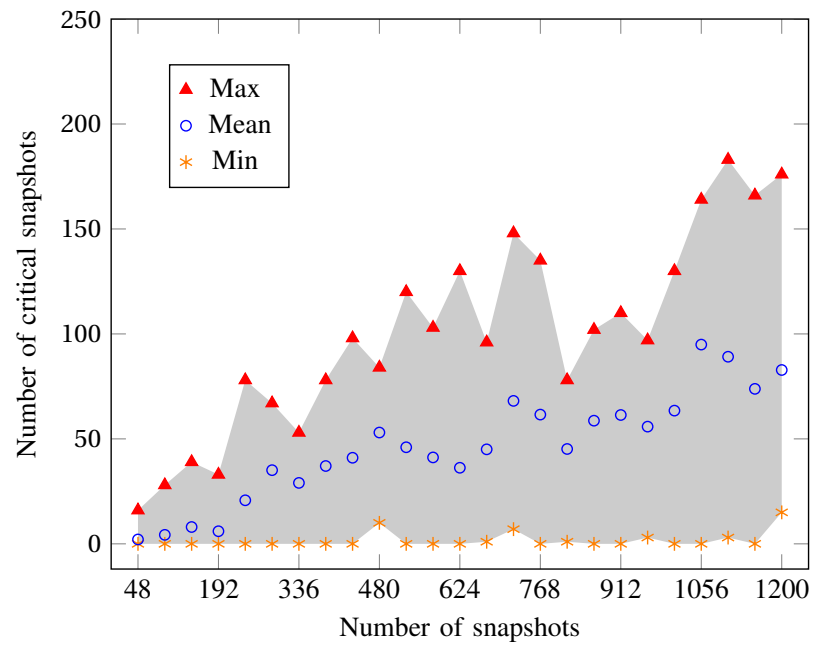

Figure 6. Vertical axis show the number of critical operating snapshots. For a given number of snapshots, 200 random samples were used to obtain min, mean and max values.

pick-ups of the system demand. This experiment shows that such temporal constraints are important factor that affect the adequacy of future power system.

\section{CONCLUSION AND FUTURE RESEARCH DIRECTIONS}

In this paper, we have proposed a stochastic optimisation approach in which the expected cost of generation plus value of lost load is minimised across a range of operating scenarios in order to provide a measure of adequacy of a power system given a large set of future operating conditions where adequacy is quantified as the proportion of demand that is met while satisfying network and generation limits. Uncertainty in demand and generation is modelled using scenarios. It is proposed that it can help a system planner to narrow down the critical future operating conditions. 


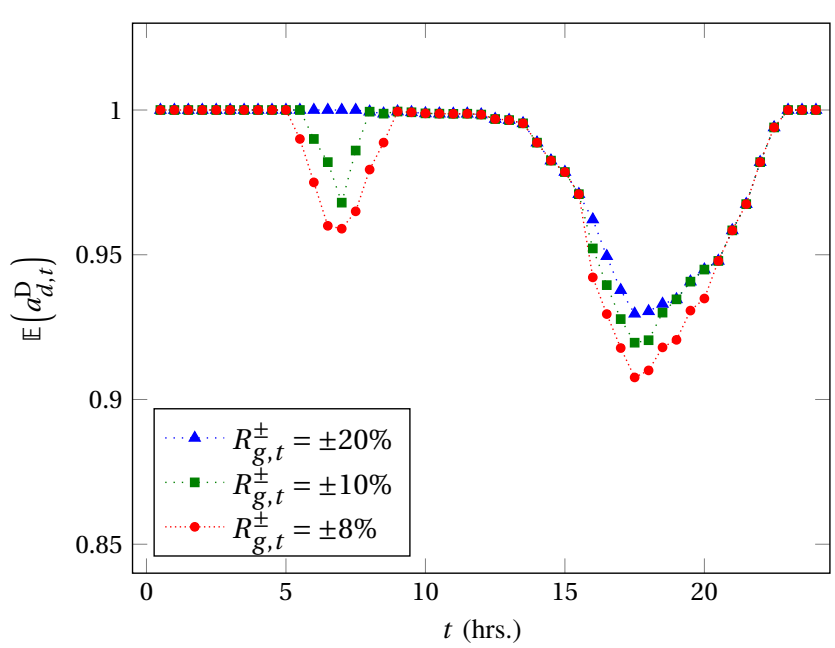

Figure 7. Effect of ramp rate constraints on the adequacy of the 39-bus system. $n^{\mathrm{D}}=10 n^{\mathrm{W}}=20$ scenarios are used for this figure.

The sensitivity of adequacy results to the selected subset of operating scenarios used in the stochastic optimisation and to generator ramp rate limits has been explored. It is concluded that care needs to be taken in the selection of operating scenarios and that ramp rates affect the apparent level of adequacy. It is suggested that the case of no ramp rate limits is equivalent to typical present day practice in transmission expansion planning in which only snapshots of states are considered and transitions between states are neglected, and that this potentially misses the need for system facilities either that provide enhanced flexibility in operation or that avoid prevention of access, due to network constraints, to sufficiently flexible resources. Furthermore, it is argued that this phenomenon will become increasingly important as the penetration of highly weather dependent renewables increases.

Future work will look at uncertainties corresponding to fuel prices, asset management and markets, and how these affect the apparent level and nature of transmission expansion required to facilitate the electricity market as distinct solely from ensuring adequate security of supply. (See, for example, [19]). Current research is focusing on the issues related to the unit commitment and $\mathrm{AC}$ modelling of the proposed approach.

\section{ACKNOWLEDGEMENTS}

The authors would like to thank Simon Gill for insightful discussions on the issues related to adequacy and security of electricity supply.

\section{REFERENCES}

[1] E. Bompard, G. Fulli, M. Ardelean, and M. Masera, "It's a bird, it's a plane, it's a...supergrid!: Evolution, opportunities, and critical issues for pan-european transmission," Power and Energy Magazine, IEEE, vol. 12, no. 2, pp. 40-50, March 2014.

[2] R. Billinton, Y. Gao, and R. Karki, "Application of a joint deterministicprobabilistic criterion to wind integrated bulk power system planning," Power Systems, IEEE Transactions on, vol. 25, no. 3, pp. 1384-1392, Aug 2010.
[3] G. Bronmo, A. Vergnol, N. Gamov, W. Bukhsh, K. Bell, A. Andreev, V. Zahov, and B. Chovikov, "Functional analysis of system development process," Tech. Rep. [Online]. Available: http://www.garpur-project.eu/deliverables

[4] W. Li, "Framework of probabilistic power system planning," Power and Energy Systems, CSEE Journal of, vol. 1, no. 1, pp. 1-8, March 2015.

[5] C. Ruiz and A. Conejo, "Robust transmission expansion planning," European Journal of Operational Research, vol. 242, no. 2, pp. 390 - 401, 2015. [Online]. Available: http://www.sciencedirect.com/science/article/pii/S0377221714008510

[6] E. Gil and I. Aravena, "Evaluating the contribution of intermittent generation to power system adequacy at different demand levels," in Power and Energy Society General Meeting (PES), 2013 IEEE, July 2013, pp. 1-5.

[7] J. Choi, T. Mount, and R. Thomas, "Transmission expansion planning using contingency criteria," Power Systems, IEEE Transactions on, vol. 22, no. 4, pp. 2249-2261, Nov 2007.

[8] B. Stott, O. Alsac, and A. Monticelli, "Security analysis and optimization," Proceedings of the IEEE, vol. 75, no. 12, pp. 1623-1644, Dec 1987.

[9] "National electricity transmission system security and quality of supply standard, version 2.2," Tech. Rep., March 52012.

[10] Balancing mechanism reporting system (bmrs). [Online] Available:http://www.bmreports.com.

[11] "Future energy scenarios," Tech. Rep., July 2015. [Online]. Available: http://fes.nationalgrid.com/

[12] J. R. Birge and F. Louveaux, Introduction to stochastic programming, ser. Springer series in operations research. New York: Springer, 1997. [Online]. Available: http://opac.inria.fr/record=b1093104

[13] J. Zhu, Optimization of Power System Operation. IEEE Press, 2009.

[14] G. Morales-Espana, J. Latorre, and A. Ramos, "Tight and compact milp formulation for the thermal unit commitment problem," in Power Energy Society General Meeting, 2015 IEEE, July 2015, pp. 1-1.

[15] "IBM ILOG CPLEX Optimizer," http://www01.ibm.com/software/integration/optimization/cplex-optimizer/, 2010.

[16] W. E. Hart, C. Laird, J.-P. Watson, and D. L. Woodruff, Pyomooptimization modeling in python. Springer Science \& Business Media, 2012, vol. 67.

[17] R. D. Zimmerman, C. E. Murillo-Sánchez, and R. J. Thomas, "Matpower: Steady-state operations, planning, and analysis tools for power systems research and education," IEEE Transactions on Power Systems, vol. 26, pp. 12-19, 2011.

[18] Balancing mechanism reporting system. [Online]. Available: http://www.bmreports.com

[19] K. Bell, "Methods and tools for planning the future power system: Issues and priorities," 2014. [Online]. Available: http://www.theiet.org/sectors/energy/resources/modellingreports/papers.cfm 